OPEN ACCESS

Edited by:

Qi Zhao,

Shenyang Aerospace University,

China

Reviewed by:

Takuichi Sato,

Niigata University, Japan

Thuy Do,

University of Leeds, United Kingdom

${ }^{*}$ Correspondence:

Liying Yu

wuyu1984@hotmail.com

Wenhong Zhang

zhangwenhong@fudan.edu.cn

†These authors have contributed equally to this work

Specialty section:

This article was submitted to Systems Microbiology,

a section of the journal

Frontiers in Microbiology

Received: 09 March 2019

Accepted: 12 July 2019

Published: 30 July 2019

Citation:

Chen J, Wu X, Zhu D, Xu M, Yu Y, Yu L and Zhang W (2019) Microbiota in Human Periodontal Abscess Revealed by $16 S$ rDNA Sequencing.

Front. Microbiol. 10:1723.

doi: 10.3389/fmicb.2019.01723

\section{Microbiota in Human Periodontal Abscess Revealed by 16S rDNA Sequencing}

\author{
Jiazhen Chen ${ }^{1+}$, Xingwen $W u^{2 \dagger}$, Danting $Z h u^{3}$, Meng $X u^{3}$, Youcheng $\mathrm{Yu}^{2}$, Liying $\mathrm{Yu}^{3 *}$ and \\ Wenhong Zhang ${ }^{1 *}$
}

${ }^{1}$ Department of Infectious Diseases, Huashan Hospital, Fudan University, Shanghai, China, ${ }^{2}$ Department of Dentistry, Zhongshan Hospital, Fudan University, Shanghai, China, ${ }^{3}$ Department of Dentistry, Huashan Hospital, Fudan University, Shanghai, China

Periodontal abscess is an oral infective disease caused by various kinds of bacteria. We aimed to characterize the microbiota composition of periodontal abscesses by metagenomic methods and compare it to that of the corresponding pocket and healthy gingival crevice to investigate the specific bacteria associated with this disease. Samples from abscess pus (AB), periodontal pocket coronally above the abscess $\mathrm{PO}$, and the gingival crevice of the periodontal healthy tooth were obtained from 20 periodontal abscess patients. Furthermore, healthy gingival crevice samples were obtained from 25 healthy individuals. Bacterial DNA was extracted and 16S rRNA gene fragments were sequenced to characterize the microbiota and determine taxonomic classification. The beta-diversity analysis results showed that the $A B$ and $P O$ groups had similar compositions. Porphyromonas gingivalis, Prevotella intermedia, and other Prevotella spp. were the predominant bacteria of human periodontal abscesses. The abundances of Filifactor alocis and Atopobium rimae were significantly higher in periodontal abscesses than in the periodontal pocket, suggesting their association with periodontal abscess formation. In conclusion, we characterized the microbiota in periodontal abscess and identified some species that are positively associated with this disease. This provides a better understanding of the components of periodontal abscesses, which will help facilitate the development of antibiotic therapy strategies.

Keywords: high-throughput sequencing, oral microbiota, periodontal abscess, 16S rDNA metagenomic, periodontal pocket

\section{INTRODUCTION}

Periodontal abscess is an acute exacerbation of chronic periodontitis, exhibiting clinical symptoms of swelling and severe pain in the gingival margin. It is defined as a localized suppurative lesion that is related to periodontal alveolar bone loss and the accumulation of pus in the gingival wall of the periodontal pocket (Herrera et al., 2000). Previously, we cultured obligate anaerobic bacteria from periodontal abscess and characterized their antimicrobial resistance profiles (Xie et al., 2014), in which the predominant obligate anaerobes were black-pigmented Prevotella. Although the results were partly in agreement with the findings of previous studies (Jaramillo et al., 2005; Herrera et al., 2014), some bacteria such as those of the genus Treponema were unculturable and some 
predominant anaerobes such as Porphyromonas gingivalis, Tannerella forsythia, and Fusobacterium spp. were less frequently cultured, due to the culture condition or suitability of medium (Xie et al., 2014). In addition, it has been reported that the therapeutic effect of antibiotic regimens on periodontal abscess is limited (Smith and Davies, 1986; Herrera et al., 2000, 2014), suggesting the complexity of associated pathogens. Recently, we used high-throughput barcoded $16 \mathrm{~S}$ rDNA sequencing to characterize the microbiota in the periodontal pocket of patients with periodontitis and compared these to those of patients with chronic obstructive pulmonary disease (COPD). As the number of different kinds of bacteria was determined in the subgingival plaque of every patient, we hypothesized that periodontal abscess is caused by a combination of microbiota, and specific pathogens might be more dominant in the abscess than in the pocket and in healthy controls suggesting a positive-association with abscess formation. Therefore, a clearer understanding of pathogens and the microbiota that cause periodontal abscess is necessary.

In this study, we used high-throughput barcoded $16 \mathrm{~S}$ rDNA sequencing technique to characterize the microbiota of periodontal abscess, the corresponding pocket, and healthy gingival crevice to investigate the specific bacteria associated with periodontal abscess in human periodontitis.

\section{MATERIALS AND METHODS}

\section{Patient Recruitment}

Forty-five participants were recruited from March 2015 to September 2015, including 20 periodontitis patients with periodontal abscess and 25 periodontal healthy individuals. Subjects with the following conditions were excluded from the study: pregnancy, use of antibiotics or anti-inflammatory drugs during the past 3 months, and administration of periodontal therapy during the last 6 months. The study was approved by the ethics committee of Huashan Hospital, Fudan University (No. KY2014-023). All participants provided signed, informed consent. The study design is shown in Supplementary Figure S1. Probing depth (PD), clinical attachment loss (CAL), and simplified oral hygiene index (OHI-S) were assessed according to World Health Organization recommendations (WHO, 1997). Periodontitis was diagnosed as previously described (Wu et al., 2017) with the presence of more than one tooth with at least one site (mesiobuccal, buccal, distobuccal, mesiolingual, lingual, and distolingual sites) with $\mathrm{PD} \geq 4 \mathrm{~mm}, \mathrm{CAL} \geq 2 \mathrm{~mm}$, and bleeding on probing. Periodontal abscess was diagnosed by a periodontal specialist based on the patients' symptomatology, clinical and radiological examination findings such as swelling and enlargement of the gingiva, history of periodontal disease, and radiograph of the alveolar bone destruction around the cementoenamel junction. Patients with periodontal abscess but without periodontitis were excluded. Periodontal tooth health was defined as $\mathrm{PD} \leq 2 \mathrm{~mm}$ and with no bleeding on probing at all six sites.

\section{Specimen Collection and Isolation of Bacterial DNA}

Three samples were collected from all the patients with abscess, including the sample of abscess pus (abscess group, $\mathrm{AB}$ ), periodontal pocket coronally above the abscess (pocket group, $\mathrm{PO}$ ), and gingival crevice of periodontal healthy tooth (patient control group, PC). Healthy teeth were also sampled from periodontally healthy individuals as the healthy control (control group, HC). The abscess samples were drained after decontamination of the mucosa. A No. 25\# sterilized paper point (Gapadent, China) was immersed into the deep area of pus for $10 \mathrm{~s}$ after drainage with a sterilized probe. The periodontal pocket and healthy gingival crevice samples were dipped with No. 25\# sterilized paper points as previously described (Wu et al., 2017). The periodontal pocket sample was not collected from one patient due to contamination with pus. All samples were stored in tris-EDTA buffer solution of pH 7.4 (Sigma, United States) in a freezer $\left(-80^{\circ} \mathrm{C}\right)$. Bacterial DNA was extracted using the QiAamp DNA Mini Kit (Qiagen, Germany), according to the manufacturer's instructions.

\section{Amplification of the 16S rDNA and Sequencing}

According to previous studies (Claesson et al., 2010; MizrahiMan et al., 2013; Jorth et al., 2014), hypervariable V3-V4 or $\mathrm{V} 4-\mathrm{V} 5$ regions are recommended to study the microbiome when using the second-generation sequencing method. It was also determined that the V4-V5 region showed the best performance among all regions. Therefore, the amplification of the V4-V5 regions of $16 \mathrm{~S}$ rDNA, library construction, index PCR, and PCR clean-up were performed as previously described (Wu et al., 2017). Equal amounts of tagged 16S rRNA gene amplicons of each sample were mixed and denatured with $0.1 \mathrm{M} \mathrm{NaOH}$. The mixed library was diluted to a final concentration of $10-20 \mathrm{pM}$ using $10 \mathrm{mM}$ tris at $\mathrm{pH}$ 8.5. Multiplexed paired-end sequencing $(2 \times 300 \mathrm{bp}$ reads $)$ of the $16 \mathrm{~S}$ rRNA amplicons was performed using a Miseq system (Illumina, San Diego, CA, United States). Image analysis and base calling were performed on the Miseq system using the MiSeq Reporter software (MSR). After demultiplexing the data and removing the reads that failed the purity filter $(\mathrm{PF}=0)$, the reads were converted to FASTQ format.

\section{Data Analyses}

The generated FASTQ files (.fastq) and quality files were acquired as raw and mapped sequence data using default settings of the QIIME2 software (version: 2018.8) (Caporaso et al., 2010). Each operational taxonomic unit (OTU) was generated with 97\% similarity cutoff using UPARSE v7.1 and chimeric sequences were identified and removed using UCHIME. The phylogenetic affiliation of each 16S rRNA gene sequence was analyzed using RDP Classifier ${ }^{1}$ based on the Silva (SSU132) 16S rRNA database using a confidence threshold of 70\% (Amato et al., 2013; Duan X.B. et al., 2017; Duan X. et al., 2017; Xu et al., 2018). The output was based on the classification of reads at several

\footnotetext{
${ }^{1}$ http://rdp.cme.msu.edu/
} 
taxonomic levels. The alpha- and beta-diversity analyses were computed from the previously constructed OTU table using Mothur software (v.1. 30.1) (Schloss et al., 2009) and weighted UniFrac (Lozupone et al., 2011) analysis. In the group level, abundance analysis was determined from rarefaction files by the Mann-Whitney test between patient and health control groups and a paired $t$-test in the self-control comparison (SPSS Statistics v20.0 and GraphPad Prism software v6.01). At the patient level, when analyzing the significantly dominant bacteria in abscess patients, any OTUs with abundance differences greater than $10 \%$ were considered significantly dominant. The minimum abundance cutoff was set at $0.1 \%$ abundance, and abundance values $<0.1 \%$ were neglected.

\section{RESULTS}

\section{Participant Characteristics}

Characteristics of the 45 enrolled subjects are listed in Table $\mathbf{1 .}$ The sex proportion and smoking status between patients and controls were not statistically different.

\section{Taxonomic Classification of the 16S rDNA Sequences}

In total, 4.1 GB raw data containing 1.70 million high-quality and classifiable reads were obtained from 84 samples. The sequencing depth was similar among the four groups as follows: $20.85 \pm 3.16 \mathrm{k}$ reads in the $\mathrm{AB}$ group, $15.50 \pm 1.93 \mathrm{k}$ reads in the PO group, $15.40 \pm 1.40 \mathrm{k}$ reads in the PC group, and

TABLE 1 | Characteristics data of the enrolled study participants.

\begin{tabular}{lcc}
\hline & Patient $(\mathbf{N}=\mathbf{2 0})$ & Health control $(\mathbf{N}=\mathbf{2 5})$ \\
\hline Gender (\%) & $11(55.0 \%)$ & $11(44.0 \%)$ \\
Male & $9(45.0 \%)$ & $14(56.0 \%)$ \\
Female & & \\
Age & $53.2(16.2)$ & $64.8(6.7)$ \\
Mean (sd) & & \\
Smoking status (\%) & $20(80.0 \%)$ \\
Non-smokera & $17(85.0 \%)$ & $5(20.0 \%)$ \\
Smoker & $3(15.0 \%)$ & $1(4.0 \%)$ \\
Former smokerb & 0 & $4(16.0 \%)$ \\
Current smoker ${ }^{\text {b }}$ & $3(15.0 \%)$ & $16.4(14.4)$ \\
Cigarettes/day, Mean (SD) & & $20(\mathrm{~N} / \mathrm{A})$ \\
Smoker & $12.3(7.5)$ & $15.5(16.5)$ \\
Former smoker & 0 & $2.5(0.5)$ \\
Current smoker & $12.3(7.5)$ & $2.8(0.7)$ \\
Periodontal Index & & $1.45(0.51)$ \\
PD, Mean (SD) & $4.8(0.7)$ & \\
CAL, Mean (SD) & $5.8(1.0)$ & \\
OHl-S, Mean (SD) & $2.43(1.12)$ & \\
\hline Non-smokers were those who & \\
\hline
\end{tabular}

${ }^{a}$ Non-smokers were those who either had never smoked or quit cigarettes at least 10 years prior to study entry. ${ }^{b}$ Former smokers were those who quit cigarettes at least 6 months but $<10$ years prior to study entry. ${ }^{\circ}$ Current smokers were currently smokers or those who quit cigarettes $<6$ months prior to study entry. $P D$, probing depth; CAL, clinical attachment loss; OHI-S, simplified oral hygiene index.
$27.31 \pm 7.21 \mathrm{k}$ reads in the HC group. Among these highquality reads, $99.41 \%$ were classified into 322 genera, belonging to 22 phyla, 38 classes, 84 orders, and 153 families. There was no significant difference in the proportion of unclassifiable sequences among the four groups based on a Kruskal-Wallis test $(P=0.186)$.

\section{Alpha-Diversity Analysis}

The alpha-diversity analysis was conducted with two indexes, namely the Shannon index implicating community diversity and the Chaol index implicating community richness (Supplementary Figure S2). Plots were generated and exported to the rarefaction curves (Aagaard et al., 2012; Supplementary Figure S3). There was no significant difference in Chaol and Shannon indexes among the four groups based on a one-way ANOVA analysis with Tukey's multiple comparisons test.

\section{Beta-Diversity Analysis}

The microbial raw OTU data were subjected to the principal coordinate analysis (PCoA) to evaluate the similarities among the four groups (Figure 1). The results showed that the samples from the $\mathrm{AB}$ and $\mathrm{PO}$ groups had similar microbiota compositions, which could be grouped into one cluster, whereas the HC group formed another cluster. The samples from the PC group could not be grouped into one cluster and were scattered in the $3 \mathrm{D}$ plot (Figure 1).

\section{Abundance Analysis}

Consistent with the results of the beta-diversity analysis, 8 of the top 10 most dominant bacteria were the same in these two groups. The genera Porphyromonas, Treponema 2, Streptococcus, Neisseria, Fusobacterium, Prevotella, Prevotella 7 and Tannerella accounted for $61 \%$ of the bacteria in the $\mathrm{AB}$ and $\mathrm{PO}$ groups, and exhibited no significant differences between the $A B$ and $P O$ groups based on a paired $t$-test (Table 3 and Supplementary Figure S4). Furthermore, based on the hierarchical clustering analysis of the four groups, 17 of the 20 (85\%) AB samples and 11 of the $19(58 \%)$ PO samples clustered together (lower cluster in Figure 2). In the PC and HC control groups, 8 of the top 10 most dominant bacteria overlapped. The genera Streptococcus, Neisseria, Bacteroides, Fusobacterium, Veillonella, Prevotella, Actinomyces, and Porphyromonas accounted for 57 and $59 \%$ of the total bacteria in the PC and HC groups, respectively (Supplementary Figure S4). Fourteen of the 20 (70\%) PC samples and 22 of the 25 (88\%) HC samples clustered together (upper cluster in Figure 2), indicating almost similar compositions between these two groups.

\section{Dominant Bacteria in Abscesses at the Patient Level}

In the $\mathrm{AB}$ group, the abundance of the most abundant OTUs in all samples did not exceed 50\% (Figure 3). The data showed that 9 of 20 samples had two OTUs with an abundance over $10 \%$ and 7 of 20 samples had three or more OTUs with an abundance over 10\%. Furthermore, 2 of 20 samples had two OTUs with an abundance over $20 \%$, including the combination 


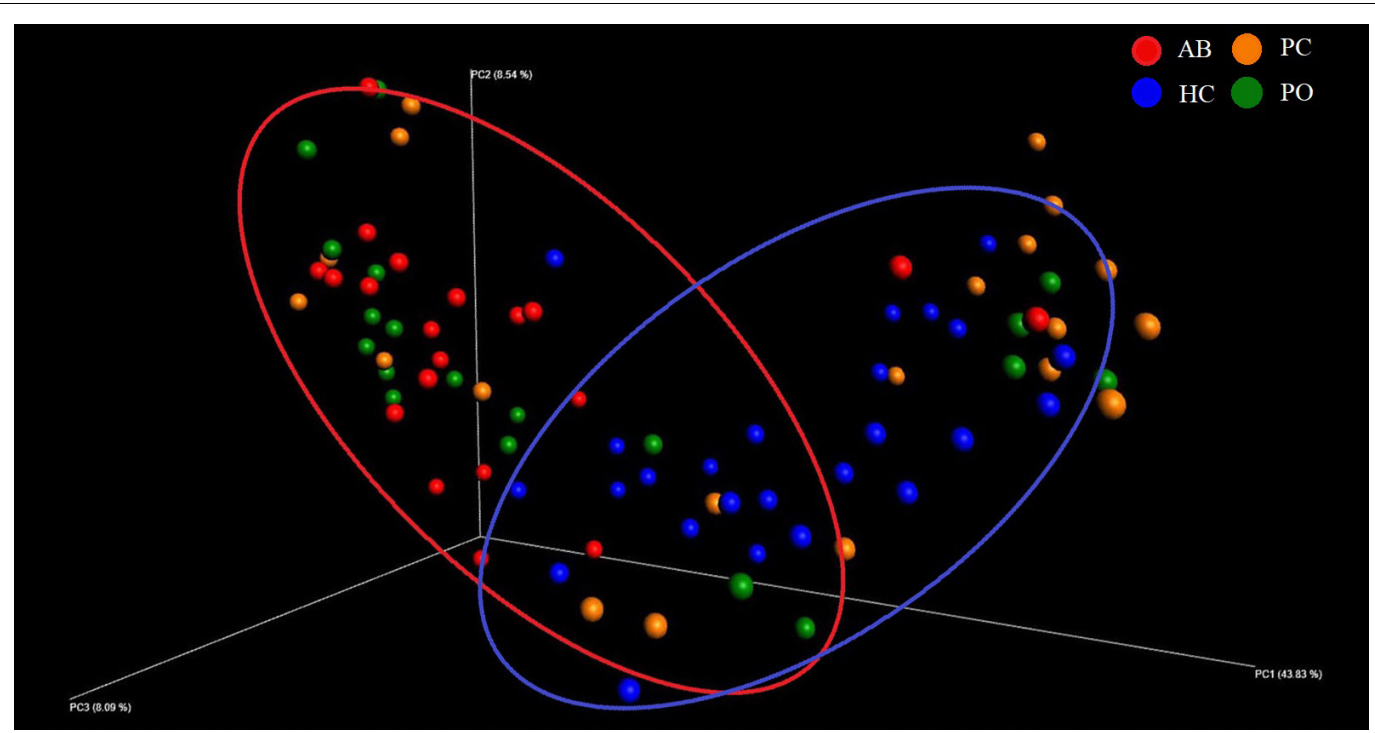

FIGURE 1 | Beta diversity analysis based on UniFrac analysis. Plots were generated using weighted UniFrac distances. Red dot represents the abscess pus (AB) group. Green dot represents the pocket (PO) group. Yellow dot represents the patient control (PC) group. Blue dot represents the healthy control (HC) group. Circles in red and blue represent different periodontal bacterial community clusters, respectively.

of Leptotrichiaceae_Unclassified (27.8\%) and P. gingivalis W83 (23.1\%) in abscess 031711C, and Lautropia_uncultured bacterium (29.5\%) and Streptococcus_uncultured bacterium (21.6\%) in abscess 052004B (Figure 3). These results suggest that disease in these patients was caused by bacterial co-infections.

To reveal the dominant bacteria involved in abscess, we compared the OTUs of the AB group with those of the PC group by performing a paired $t$-test at the group level. The results showed that the abundance of 6 OTUs was significantly higher in the $\mathrm{AB}$ than in the $\mathrm{PC}$ group (Supplementary Table S1). However, except $P$. gingivalis W83, other classical opportunistic bacteria causing periodontal abscess were not significantly different based on this analysis. Furthermore, the OTUs, except $P$. gingivalis W83, which was relatively high in the $\mathrm{AB}$ group, were low in average abundance (Supplementary Table S1), suggesting that group comparison is not ideal for the analysis of dominant bacteria.

Considering the heterogeneity of dominant opportunistic bacteria in different patients, we performed a direct bacterial abundance comparison between the $\mathrm{AB}$ and $\mathrm{PC}$ groups at the patient level, and the bacteria with abundance differences $>10 \%$ between the $\mathrm{AB}$ and $\mathrm{PC}$ groups were identified as significantly dominant bacteria (Table 2 and Supplementary Table S2). In total, 19 OTUs including $P$. gingivalis W83 (8/20, 40\%), Prevotella spp. (3/20, 15\%), Prevotella intermedia (2/20, 10\%), P. gingivalis TDC60 (1/20,5\%), and Prevotella heparinolytica $(1 / 20,5 \%)$, were found to be significantly dominant in the $\mathrm{AB}$ group compared with abundances in the $\mathrm{PC}$ group in the corresponding number of patients (Table 2). Additionally, 20 OTUs, including Streptococcus spp. (6/20, 30\%), Actinomyces spp. (3/20, 15\%), Lautropia spp. (3/20, 15\%), Neisseria spp. (3/20, $15 \%)$, Veillonella spp. (3/20, 15\%), Fusobacterium spp. (2/20, $10 \%)$, P. intermedia (2/20, $10 \%)$, and Bacteroides neonati $(2 / 20$,
$10 \%$ ), were found to be significantly more dominant in the PC group than in $\mathrm{AB}$ group (Table 2). Interestingly, $P$. intermedia was identified as significantly dominant in the $\mathrm{AB}$ group of some patients and the PC group of other patients, indicating that it might have a heterogeneous function in abscess formation in different populations.

\section{Specific Bacteria in Abscess Compared With Those in Pockets at the Group Level}

At the group level, a comparison between the $\mathrm{AB}$ and PO groups revealed specific bacteria associated with acute disease. The abundance of bacteria in these two groups was highly similar. At the genus level, only Filifactor and Atopobium exhibited significantly higher abundance in the AB group, whereas nine genera presented significantly lower in abundance in the $\mathrm{AB}$ group than in the PO group (Table 3 ). Similarly, 3 OTUs including Filifactor alocis and Atopobium rimae presented significantly higher abundance in the $\mathrm{AB}$ group, suggesting that they might function in periodontal abscess formation. Moreover, 4 OTUs exhibited significantly lower abundance in the $\mathrm{AB}$ group than in the $\mathrm{PO}$ group (Table 3). However, all 4 OTUs were not accurately classified to the species level.

\section{Bacteria Associated With Abscess at the Group Level}

At the group level, a comparison between the $\mathrm{AB}$ and $\mathrm{HC}$ groups was made in the present study. At the genus level, 24 genera including Porphyromonas, Treponema 2, Tannerella, Filifactor, Parvimonas, and Prevotella 1 were significantly more abundant in the AB group than in the HC group (Supplementary Table S3). Moreover, 25 genera including Streptococcus, Neisseria, 
TABLE 2 | The Dominant OTUs in AB group compared to PC group at patient level.

\section{Greater than $10 \%$ higher in $\mathrm{AB}$ group than in $\mathrm{PC}$ group}

Porphyromonas gingivalis W83

Prevotella_uncultured bacterium

Prevotella intermedia

Streptococcus constellatus subsp. constellatus

Fusobacterium_uncultured bacterium

Porphyromonas_Unclassified

Porphyromonas gingivalis TDC60

Prevotella heparinolytica

[Eubacterium] brachy

Fusobacterium_uncultured organism

Leptotrichiaceae_Unclassified

Porphyromonas_uncultured organism

Prevotella 1_Unclassified

Rikenellaceae RC9 gut group_uncultured bacterium

Streptococcus_uncultured bacterium

Tannerella_Unclassified

Treponema 2_Unclassified

Treponema 2_uncultured Treponema sp.

Treponema 2_uncultured bacterium

Greater than $\mathbf{1 0} \%$ lower in $\mathrm{AB}$ group than in $\mathrm{PC}$ group

$1(5.0 \%)$

Streptococcus_uncultured bacterium

Actinomyces_uncultured bacterium

Lautropia_uncultured bacterium

Bacteroides neonati

Fusobacterium_uncultured bacterium

Porphyromonas gingivalis W83

The dominant different abundance of OTU was set as 10\%.

Veillonella, Capnocytophaga, Actinomyces, Selenomonas 3, and Prevotella 2 were significantly less abundant in the $\mathrm{AB}$ group than in the HC group (Supplementary Table S3). At the OTU level, 28 OTUs including $P$. gingivalis, Treponema 2 spp., P. intermedia, F. alocis, and T. forsythia exhibited significantly higher abundance in the $A B$ group than in the HC group. In contrast, 22 OTUs including Streptococcus spp., Veillonella spp., Actinomyces spp., and Neisseria spp. showed less abundance in the $\mathrm{AB}$ group than in the $\mathrm{HC}$ group (Table 4).

Porphyromonas gingivalis and $P$. intermedia were found to be dominant in the $A B$ group at the patient level, and their abundance was significantly higher in the $A B$ group than in the HC group at the group level. Additionally, Prevotella spp. was identified to be the dominant species in the abscesses of a few patients, but its abundance was not significantly different 
TABLE 3 | Specific bacteria of significant difference in AB compared with PO group based on a paired $t$-test at group level.

\begin{tabular}{|c|c|c|c|}
\hline Genus/OTUs & $A B($ Mean $\pm S E, \%)$ & $\mathrm{PO}$ (Mean $\pm \mathrm{SE}, \%$ ) & $p$-value \\
\hline \multicolumn{4}{|l|}{ Higher in $A B$ in Genus level } \\
\hline Filifactor & $2.05 \pm 0.40$ & $1.06 \pm 0.26$ & 0.009 \\
\hline \multicolumn{4}{|l|}{ Lower in $A B$ in Genus level } \\
\hline Leptotrichia & $0.15 \pm 0.05$ & $0.96 \pm 0.25$ & 0.005 \\
\hline Prevotella 2 & $0.06 \pm 0.02$ & $0.52 \pm 0.14$ & 0.009 \\
\hline Flexilinea & $0.09 \pm 0.04$ & $0.44 \pm 0.16$ & 0.048 \\
\hline Eikenella & $0.02 \pm 0.01$ & $0.23 \pm 0.09$ & 0.037 \\
\hline F0332 & $0.03 \pm 0.02$ & $0.12 \pm 0.05$ & 0.029 \\
\hline Bergeyella & $0.04 \pm 0.02$ & $0.11 \pm 0.04$ & 0.012 \\
\hline Filifactor alocis ATCC 35896 & $1.88 \pm 0.43$ & $0.77 \pm 0.24$ & 0.007 \\
\hline Atopobium rimae & $0.68 \pm 0.31$ & $0.00 \pm 0.00$ & 0.039 \\
\hline \multicolumn{4}{|l|}{ Lower in $A B$ in OTU level } \\
\hline Capnocytophaga_uncultured bacterium & $0.26 \pm 0.11$ & $1.82 \pm 0.67$ & 0.027 \\
\hline Veillonella_uncultured bacterium & $0.20 \pm 0.09$ & $1.42 \pm 0.61$ & 0.040 \\
\hline Desulfobulbus_Unclassified & $0.24 \pm 0.13$ & $0.61 \pm 0.22$ & 0.019 \\
\hline Leptotrichia_uncultured bacterium & $0.12 \pm 0.04$ & $0.55 \pm 0.19$ & 0.034 \\
\hline
\end{tabular}

Abundance cutoff was set at $0.1 \%$, some below $0.1 \%$ were not shown in the table.

between the $\mathrm{AB}$ and $\mathrm{HC}$ groups and it could not be classified at the species level. In contrast, Streptococcus spp., Actinomyces spp., Lautropia spp., Neisseria spp., and Veillonella spp. were the dominant species in the PC group compared with those in the $\mathrm{AB}$ group at the patient level, and their abundance was significantly lower in the $A B$ group than in the $\mathrm{HC}$ group at the group level.

\section{Specific Bacteria Associated With Periodontitis at the Group Level}

We also compared the bacterial abundance between the $\mathrm{PO}$ and $\mathrm{HC}$ groups at the group level to determine if our data were consistent with well-known periodontitisassociated bacteria. At the genus level, 23 genera including genus Porphyromonas, Treponema 2, Tannerella, Fretibacterium, Prevotella 1, Filifactor, Dialister, and Desulfobulbus were significantly higher in the PO group than in the $\mathrm{HC}$ group, whereas 17 genera including Streptococcus, Bacteroides, Veillonella, Bergeyella, and Kingella were lower in the PO group than in the HC group (Supplementary Table S4). At the OTU level, the abundance of 29 OTUs including P. gingivalis, P. intermedia, Treponema spp., T. forsythia, $F$. alocis, and $P$. heparinolytica were higher in the PO group than in the HC group. The results were partly concordant with a previous study about the periodontal red and orange complex (Newman et al., 2015). In contrast, the abundance of 23 OTUs including Streptococcus spp. and
B. neonati were lower in the PO group than in the HC group (Supplementary Table S5).

\section{DISCUSSION}

Oral periodontal abscess is an oral infective, painful disease that can spread (Yoneda et al., 2011; Herrera et al., 2014; Sato et al., 2016), and it is a valuable potential sign of undiagnosed type 2 diabetes (Alagl, 2017). Several studies have identified the dominant microbiota by culture-based diagnostic methods (Newman and Sims, 1979; Jaramillo et al., 2005; Xie et al., 2014). In the present study, considering the heterogeneity of dominant opportunistic bacteria in different patients, a patient level analysis between abscess and healthy periodontium was made, which showed that $P$. gingivalis, and Prevotella spp. including $P$. intermedia were found to be dominant in the abscess of some patients compared to those of healthy periodontium based on 16S rDNA metagenomic sequencing. Compared to the findings of our previous culture-based study, our study confirmed that Prevotella spp., and especially $P$. intermedia, is the dominant species in human periodontal abscess (Xie et al., 2014).

However, this study differs from the traditional culture-based method in the following aspects. First, the culture method usually detects the most abundant bacterium, but the second or the third most abundant bacteria can be neglected. For example, in abscess samples from two patients, $P$. gingivalis W83 was the most dominant bacterium, and the abundance was 39.4 and 


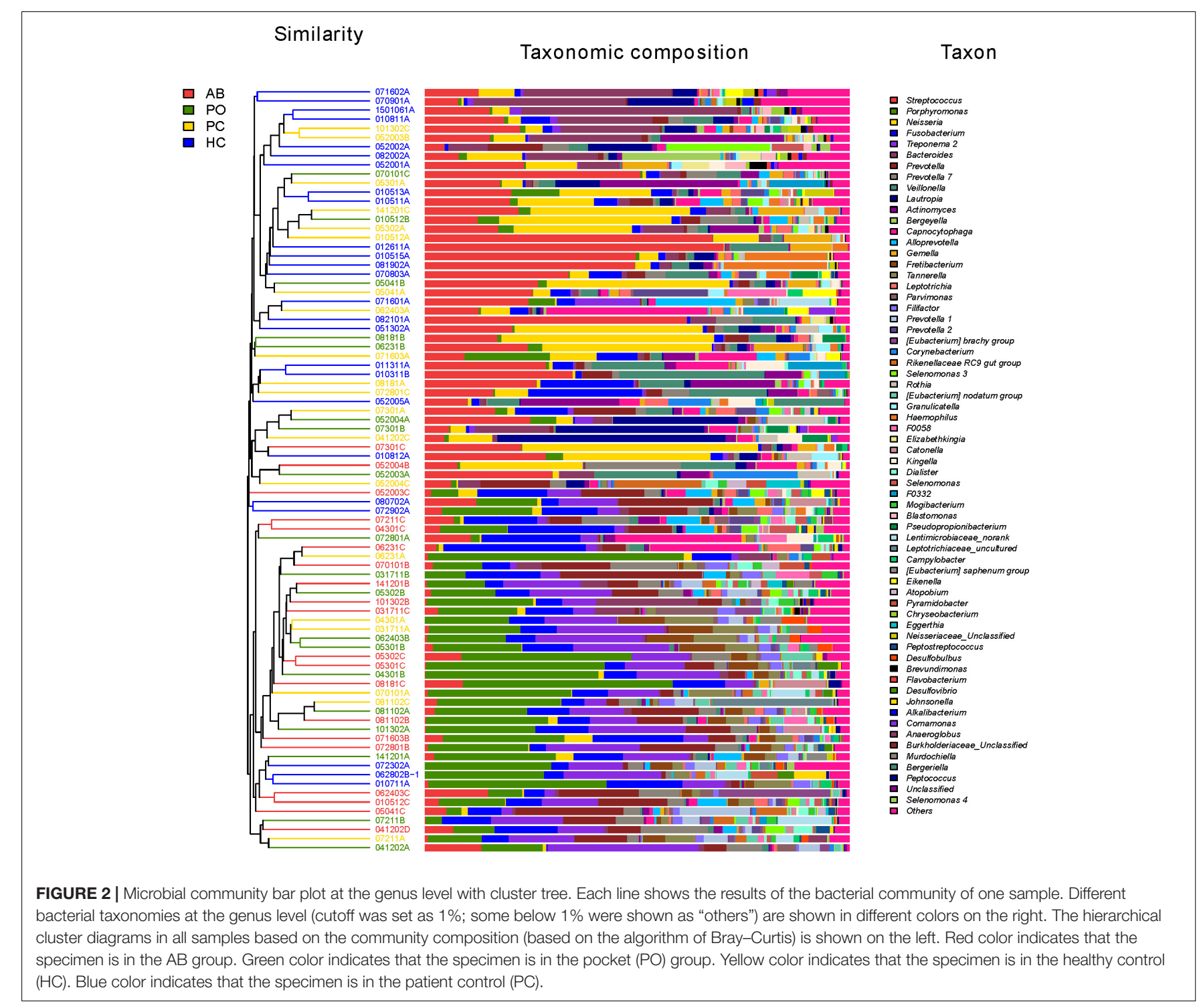

$28.5 \%$, respectively. Furthermore, the second highest abundant bacterium in both samples was Fusobacterium spp., for which abundance was only 12.3 and $17.9 \%$, respectively, which could be neglected in the culture method. Second, the metagenomic sequencing method can detect unculturable bacteria and is not restricted to medium selectivity or addictive antibiotics. For example, in one abscess sample, the dominant bacterium was Treponema 2 spp. (Table 2), which is unculturable. In addition, the major dominant bacterium identified in the present study was $P$. gingivalis, which was not detected in some by the culture method (Newman and Sims, 1979; Jaramillo et al., 2005; Xie et al., 2014). This might be attributed to medium selectivity or addition of selective antibiotics that inhibit this bacterium.

Porphyromonas gingivalis is a member of periodontal red complex (Socransky et al., 1998; Newman et al., 2015), which is the most predominant bacterial cluster detected in subgingival plaque, and can induce the production of interleukin-1 in macrophages (Saito et al., 1997) and trigger polyclonal B-cell activation (Champaiboon et al., 2000) associated with bleeding on probing and alveolar bone loss (Socransky et al., 1998). Moreover, it might be associated with several general dysfunctions including cardiovascular disease (Kozarov et al., 2005), rheumatoid arthritis (Berthelot and Le Goff, 2010), Alzheimer's disease (Dominy et al., 2019), and conception in women (Paju et al., 2017). Prevotella spp. including P. intermedia is a member of the periodontal orange complex (Newman et al., 2015), the second most predominant bacterial cluster detected in subgingival plaque, in addition to being recognized pathogens of periodontal infection. In a study by Jaramillo et al. (2005), the most frequent subgingival bacterium was Fusobacterium spp. (75\%), followed by $P$. intermedia and $P$. nigrescens (60\%), as well as $P$. gingivalis (51\%). In partial agreement of the results of our previous study that $P$. intermedia is the most prevalent bacterium in periodontal abscess (Xie et al., 2014), in the present study, the second and third most dominant bacteria were Prevotella spp. and P. intermedia (25\%). 

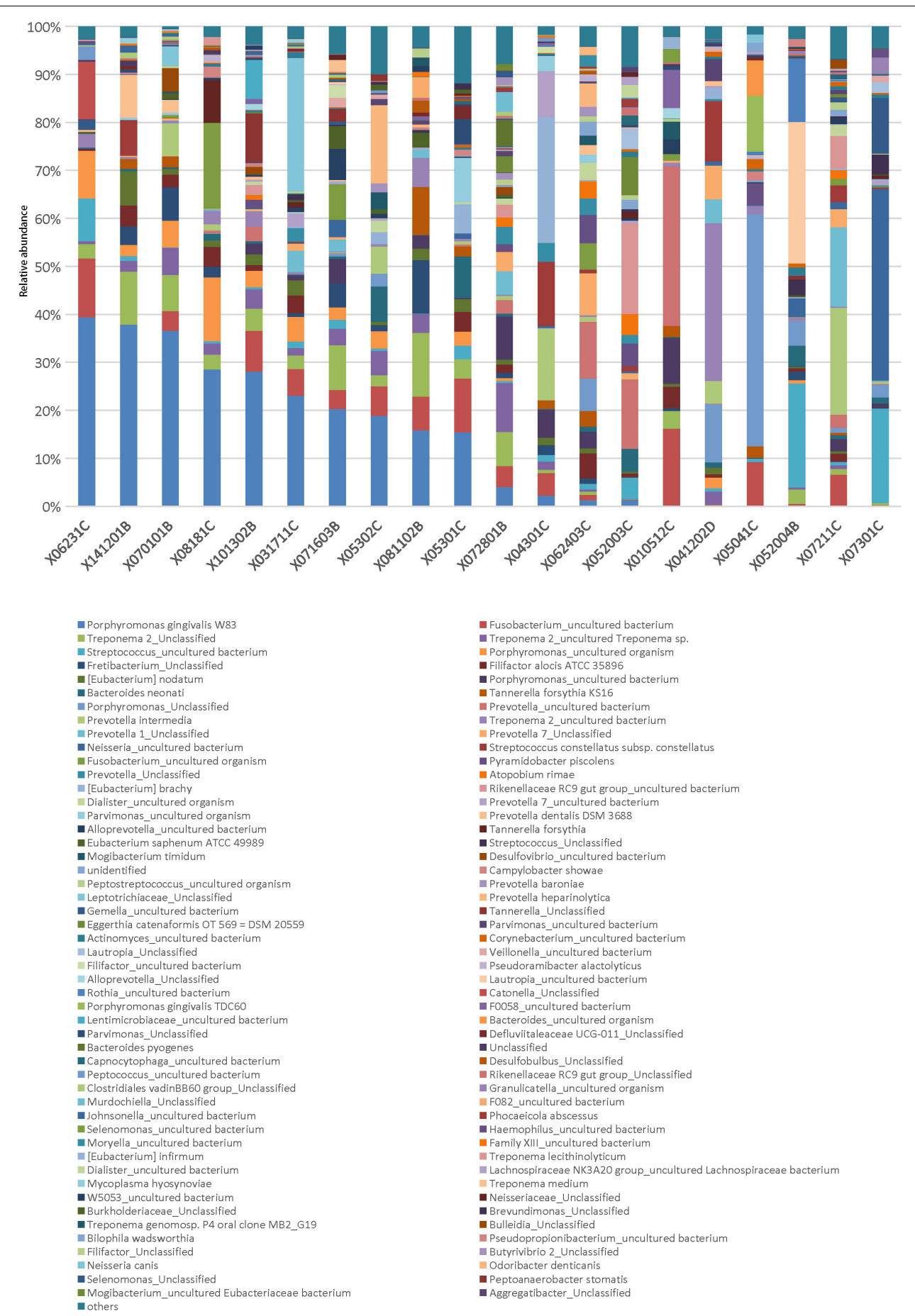

FIGURE 3 | Relative abundance of genera in the AB group. Each column represents a sample in the AB group. The genera were sorted in descending order of average abundance in abscess samples. Different bacterial taxonomies at the genus level are shown in different colors on the bottom. Abundance cutoff in this bar graph was set at 1\%; some below $1 \%$ were shown as "others."

Like brain, lung, and pyogenic liver abscesses, which are caused by multiple kinds of bacteria (Brook, 2009; Webb et al., 2014; Yazbeck et al., 2014), periodontal abscess is more complex than previously thought. In the present study, seven of 20 samples had three or more OTUs with an abundance greater than $10 \%$, and most OTUs were opportunistic bacteria, suggesting pathogen heterogeneity and bacterial co-infection in periodontal abscess diseases. Abscess occurs in a site that inhabits multiple normal and opportunistic bacteria, which are symbiotic and promote abscess formation (Newman et al., 2015). The significantly 
TABLE 4 | Mean relative abundance of OTUs with significant statistical difference between AB and HC groups at group level.

OTUs

$A B($ Mean \pm SE, \%)

HC (Mean \pm SE, \%)

p-value

\section{Higher in AB}

Prevotella intermedia

Treponema 2_uncultured Treponema sp.

Prevotella 1_Unclassified
Porphyromonas gingivalis W83

Treponema 2_Unclassified

Treponema 2_uncultured bacterium

Porphyromonas_uncultured organism

Streptococcus constellatus subsp. constellatus

[Eubacterium] brachy

Fretibacterium_Unclassified

Filifactor alocis ATCC 35896

Rikenellaceae RC9 gut group_uncultured bacterium

[Eubacterium] nodatum

Tannerella forsythia KS16

Prevotella heparinolytica

Eubacterium saphenum ATCC 49989

Parvimonas_uncultured bacterium

Defluviitaleaceae UCG-011_Unclassified

Rikenellaceae RC9 gut group_Unclassified

Clostridiales vadinBB60 group_Unclassified

Pseudoramibacter alactolyticus

Phocaeicola abscessus

Moryella_uncultured bacterium

Family XIII_uncultured bacterium

Mycoplasma hyosynoviae

W5053_uncultured bacterium

Flexilinea_Unclassified

Bulleidia_Unclassified

\section{Lower in $A B$}

Streptococcus_uncultured bacterium

Streptococcus_Unclassified

Veillonella_uncultured bacterium

Actinomyces_uncultured bacterium

Neisseria_Unclassified

Capnocytophaga_uncultured bacterium

Prevotella 7_uncultured bacterium

Lautropia_uncultured bacterium

Gemella_uncultured bacterium

Leptotrichia_uncultured bacterium

F0332_uncultured bacterium

Granulicatella_uncultured bacterium

Selenomonas 3_uncultured bacterium

Blastomonas_uncultured bacterium

Ruminococcaceae UCG-014_uncultured bacterium

Kingella_uncultured bacterium

Delftia_Unclassified

Flavobacterium_Unclassified

Brevundimonas_uncultured bacterium

Aggregatibacter_uncultured bacterium

Ralstonia_Unclassified

Lachnoanaerobaculum uncultured bacterium

$$
\begin{aligned}
& 13.64 \pm 3.25 \\
& 3.91 \pm 0.86 \\
& 2.94 \pm 1.31 \\
& 2.74 \pm 1.62 \\
& 2.63 \pm 0.81 \\
& 2.25 \pm 0.58 \\
& 1.98 \pm 0.93 \\
& 1.95 \pm 1.32 \\
& 1.90 \pm 0.64 \\
& 1.79 \pm 0.42 \\
& 1.77 \pm 0.87 \\
& 1.53 \pm 0.99 \\
& 1.37 \pm 0.37 \\
& 1.34 \pm 0.52 \\
& 1.10 \pm 0.83 \\
& 0.59 \pm 0.27 \\
& 0.46 \pm 0.23 \\
& 0.29 \pm 0.14 \\
& 0.23 \pm 0.07 \\
& 0.22 \pm 0.08 \\
& 0.19 \pm 0.09 \\
& 0.18 \pm 0.09 \\
& 0.15 \pm 0.11 \\
& 0.15 \pm 0.05 \\
& 0.12 \pm 0.06 \\
& 0.12 \pm 0.07 \\
& 0.11 \pm 0.04 \\
& 0.10 \pm 0.09 \\
& 3.42 \pm 1.40 \\
& 0.56 \pm 0.25 \\
& 0.29 \pm 0.12 \\
& 0.44 \pm 0.10 \\
& 0.11 \pm 0.06 \\
& 0.26 \pm 0.11 \\
& 0.79 \pm 0.49 \\
& 1.52 \pm 1.47 \\
& 0.81 \pm 0.58 \\
& 0.12 \pm 0.04 \\
& 0.01 \pm 0.01 \\
& 0.06 \pm 0.02 \\
& 0.06 \pm 0.05 \\
& 0.00 \pm 0.00 \\
& 0.00 \pm 0.00 \\
& 0.00 \pm 0.00 \\
& 0.00 \pm 0.00 \\
& 0.04 \pm 0.02 \\
& 0.01 \pm 0.01 \\
& 0.00 \pm 0.00 \\
& 0.00 \pm 0.00 \\
& 0.01 \pm 0.00
\end{aligned}
$$

\begin{tabular}{|c|c|}
\hline $0.09 \pm 0.04$ & $<0.001$ \\
\hline $0.75 \pm 0.30$ & $<0.001$ \\
\hline $0.17 \pm 0.07$ & 0.022 \\
\hline $0.46 \pm 0.19$ & 0.003 \\
\hline $0.00 \pm 0.00$ & $<0.001$ \\
\hline $0.08 \pm 0.03$ & $<0.001$ \\
\hline $0.09 \pm 0.05$ & 0.006 \\
\hline $0.15 \pm 0.07$ & 0.018 \\
\hline $0.20 \pm 0.09$ & 0.004 \\
\hline $0.05 \pm 0.03$ & $<0.001$ \\
\hline $0.01 \pm 0.01$ & 0.003 \\
\hline $0.00 \pm 0.00$ & 0.006 \\
\hline $0.03 \pm 0.02$ & $<0.001$ \\
\hline $0.05 \pm 0.03$ & 0.005 \\
\hline $0.00 \pm 0.00$ & 0.022 \\
\hline $0.02 \pm 0.01$ & 0.002 \\
\hline $0.02 \pm 0.01$ & $<0.001$ \\
\hline $0.03 \pm 0.02$ & 0.002 \\
\hline $0.00 \pm 0.00$ & $<0.001$ \\
\hline $0.01 \pm 0.01$ & 0.003 \\
\hline $0.00 \pm 0.00$ & 0.016 \\
\hline $0.00 \pm 0.00$ & 0.001 \\
\hline $0.00 \pm 0.00$ & 0.002 \\
\hline $0.01 \pm 0.01$ & 0.001 \\
\hline $0.00 \pm 0.00$ & 0.006 \\
\hline $0.00 \pm 0.00$ & 0.009 \\
\hline $0.01 \pm 0.00$ & 0.003 \\
\hline $0.00 \pm 0.00$ & 0.009 \\
\hline $13.21 \pm 2.24$ & $<0.001$ \\
\hline $6.23 \pm 1.78$ & $<0.001$ \\
\hline $4.35 \pm 1.38$ & $<0.001$ \\
\hline $3.20 \pm 1.21$ & 0.001 \\
\hline $2.98 \pm 1.16$ & 0.001 \\
\hline $2.94 \pm 0.96$ & $<0.001$ \\
\hline $2.03 \pm 0.60$ & 0.005 \\
\hline $1.68 \pm 0.66$ & 0.005 \\
\hline $1.63 \pm 0.47$ & $<0.001$ \\
\hline $1.45 \pm 0.34$ & $<0.001$ \\
\hline $1.13 \pm 0.60$ & 0.005 \\
\hline $0.81 \pm 0.18$ & $<0.001$ \\
\hline $0.81 \pm 0.28$ & 0.011 \\
\hline $0.52 \pm 0.23$ & 0.006 \\
\hline $0.45 \pm 0.23$ & 0.006 \\
\hline $0.44 \pm 0.20$ & $<0.001$ \\
\hline $0.29 \pm 0.13$ & 0.011 \\
\hline $0.25 \pm 0.08$ & 0.011 \\
\hline $0.24 \pm 0.07$ & 0.002 \\
\hline $0.22 \pm 0.11$ & 0.012 \\
\hline $0.18 \pm 0.06$ & 0.012 \\
\hline $0.14 \pm 0.05$ & 0.009 \\
\hline
\end{tabular}

Abundance cutoff was set at $0.1 \%$, some below $0.1 \%$ were not shown in the table. 
dominant bacteria in the abscess were also diverse in different patients and the difference between the abscess and the pocket remains unknown.

To the best of our knowledge, the bacteria in the periodontal abscess and periodontal pocket were compared for the first time. Periodontal abscess might represent acute exacerbation of periodontitis that is favored by changes in the subgingival microbiota, with an increase in bacterial virulence or a decrease in host defense (Herrera et al., 2014), resulting in the disruption of chronic phase (pocket) homeostasis and conversion to the acute phase (abscess). It is noteworthy that only two OTUs, $F$. alocis and $A$. rimae, were significantly higher in abundance in the $\mathrm{AB}$ group than in the $\mathrm{PO}$ group at the group level, indicating that they could be associated with the exacerbation of chronic periodontitis to acute periodontal abscess, although bacterial abundance and diversity were highly similar between the $\mathrm{AB}$ and $\mathrm{PO}$ groups. F. alocis is a Grampositive anaerobic rod, which is now suggested to be a new periodontal pathogen (Schlafer et al., 2010; Aruni et al., 2015; Camelo-Castillo et al., 2015) with unique properties such as resistance to oxidative stress (Aruni et al., 2011), the ability to cause chronic inflammation (Fine et al., 2013), and the capacity to trigger apoptosis of gingival epithelial cells (Moffatt et al., 2011). In the present study, consistent with the findings of previous studies that $F$. alocis is positively associated with periodontitis, $F$. alocis was found to be more abundant in periodontal pockets than in the healthy periodontium. Furthermore, it was more abundant in periodontal abscess than in the pocket, suggesting that it is a potential, acute abscessrelated, periodontal pathogen. A. rimae is an anaerobic, Grampositive, rod-shaped bacterium, which has been suggested to be an endodontic abscess-related microorganism (Tennert et al., 2014; George et al., 2016). In the present study, A. rimae was found to be more significantly abundant in the abscess than in the pocket and healthy periodontium of the same patient. However, it has been reported that A. rimae is more prevalent in healthy subjects (Kumar et al., 2003), suggesting its role in periodontitis formation, which is complex and requires further study.

The bacteria associated with periodontitis have been well investigated previously (Liu et al., 2012; Wang et al., 2013). In the present study, the finding that the abundance of 29 OTUs including $P$. gingivalis, $P$. intermedia, T. forsythia, and $F$. alocis was higher in the PO group than in the HC group was largely in agreement with the findings of previous studies (Liu et al., 2012; Wang et al., 2013). These data further strengthen the reliability of this study to investigate the opportunistic pathogens and dominant microbiota associated with periodontal abscess.

Meanwhile, there were some limitations to our study. First, only the V4-V5 region, and not the full-length gene, was sequenced, which might result in some unclassified OTUs like Streptococcus_Unclassified at the species level. Second, different bacterial databases could lead to differences in detected species, which requires further comparisons with previously published studies to confirm the suitability of the present research. Third, the present study did not quantify the bacterial loads in samples, and quantitative research will be more helpful in unraveling the relationship between the severity of periodontal abscess and certain bacteria.

In conclusion, we used $16 \mathrm{~S}$ rRNA-based metagenomics to characterize the bacterial profile of periodontal abscess in humans and compared it with the corresponding periodontal pocket and healthy periodontium. The results showed that the bacterial composition of periodontal abscess is more complex and mainly involves bacterial co-infections. Further, $P$. gingivalis, $P$. intermedia, and Prevotella spp. were the predominant bacteria in human periodontal abscesses. Two species, F. alocis and A. rimae, were found to be positively associated with abscess formation, although their bacterial abundance and diversity in periodontal abscess and periodontal pockets were highly similar. Recognition of the bacterial profile of periodontal abscess might reveal new strategies for the diagnosis, surveillance, and treatment of periodontal abscess, including the accurate use of antibiotics and probiotics.

\section{DATA AVAILABILITY}

All sequencing data were uploaded and deposited to the SRA database with project number PRJNA547446.

\section{ETHICS STATEMENT}

This study was approved by the Ethics Committee of the Huashan Hospital, Fudan University (No. KY2014-023).

\section{AUTHOR CONTRIBUTIONS}

JC and XW conceived and designed the study, acquired, analyzed, and interpreted the data, and drafted and critically revised the manuscript. DZ, MX, and YY acquired the data and critically revised the manuscript. LY and $\mathrm{WZ}$ conceived and designed the study, analyzed and interpreted the data, and drafted and critically revised the manuscript. All authors approved the final manuscript and agreed to be accountable for all aspects of the work.

\section{FUNDING}

This work was supported by the National Natural Science Foundation of China (81471987).

\section{SUPPLEMENTARY MATERIAL}

The Supplementary Material for this article can be found online at: https://www.frontiersin.org/articles/10.3389/fmicb.2019. 01723/full\#supplementary-material 


\section{REFERENCES}

Aagaard, K., Riehle, K., Ma, J., Segata, N., Mistretta, T. A., Coarfa, C., et al. (2012). A metagenomic approach to characterization of the vaginal microbiome signature in pregnancy. PLoS One 7:e36466. doi: 10.1371/journal.pone.00 36466

Alagl, A. S. (2017). Periodontal abscess as a possible oral clinical sign in the diagnosis of undiagnosed diabetes mellitus of elderly in a dental clinic set up - a 7-year cross-sectional study. J. Investig. Clin. Dent. 8:e12217. doi: 10.1111/ jicd. 12217

Amato, K. R., Yeoman, C. J., Kent, A., Righini, N., Carbonero, F., Estrada, A., et al. (2013). Habitat degradation impacts black howler monkey (Alouatta pigra) gastrointestinal microbiomes. ISME J. 7, 1344-1353. doi: 10.1038/ismej. 2013.16

Aruni, A. W., Mishra, A., Dou, Y., Chioma, O., Hamilton, B. N., and Fletcher, H. M. (2015). Filifactor alocis-a new emerging periodontal pathogen. Microb. Infect. 17, 517-530. doi: 10.1016/j.micinf.2015.03.011

Aruni, A. W., Roy, F., and Fletcher, H. (2011). Filifactor alocis has virulence attributes that can enhance its persistence under oxidative stress conditions and mediate invasion of epithelial cells by Porphyromonas gingivalis. Infect. Immun. 79, 3872-3886. doi: 10.1128/IAI.05631-11

Berthelot, J. M., and Le Goff, B. (2010). Rheumatoid arthritis and periodontal disease. Joint Bone Spine 77, 537-541. doi: 10.1016/j.jbspin.2010. 04.015

Brook, I. (2009). Microbiology and antimicrobial treatment of orbital and intracranial complications of sinusitis in children and their management. Int. J. Pediatr. Otorhinolaryngol. 73, 1183-1186. doi: 10.1016/j.ijporl.2009.01.020

Camelo-Castillo, A., Novoa, L., Balsa-Castro, C., Blanco, J., Mira, A., and Tomas, I. (2015). Relationship between periodontitis-associated subgingival microbiota and clinical inflammation by $16 \mathrm{~S}$ pyrosequencing. J. Clin. Periodontol. 42, 1074-1082. doi: $10.1111 /$ jcpe. 12470

Caporaso, J. G., Kuczynski, J., Stombaugh, J., Bittinger, K., Bushman, F. D., Costello, E. K., et al. (2010). QIIME allows analysis of high-throughput community sequencing data. Nat. Methods 7, 335-336. doi: 10.1038/nmeth. f.303

Champaiboon, C., Yongvanitchit, K., Pichyangkul, S., and Mahanonda, R. (2000). The immune modulation of B-cell responses by Porphyromonas ginginvalis and interleukin-10. J. Periodontol. 71, 468-475. doi: 10.1902/jop.2000.71.3.468

Claesson, M. J., Wang, Q., O'Sullivan, O., Greene-Diniz, R., Cole, J. R., Ross, R. P., et al. (2010). Comparison of two next-generation sequencing technologies for resolving highly complex microbiota composition using tandem variable $16 \mathrm{~S}$ rRNA gene regions. Nucleic Acids Res. 38:e200. doi: 10.1093/nar/gkq873

Dominy, S. S., Lynch, C., Ermini, F., Benedyk, M., Marczyk, A., Konradi, A., et al. (2019). Porphyromonas gingivalis in Alzheimer's disease brains: evidence for disease causation and treatment with small-molecule inhibitors. Sci. Adv. 5:eaau3333. doi: 10.1126/sciadv.aau3333

Duan, X.-B., Wu, T.-X., Guo, Y.-C., Zhou, X.-D., Lei, Y.-L., Xu, X., et al. (2017). Marginal bone loss around non-submerged implants is associated with salivary microbiome during bone healing. Int. J. Oral Sci. 9, 95-103. doi: 10.1038/ijos. 2017.18

Duan, X., Wu, T., Xu, X., Chen, D., Mo, A., Lei, Y., et al. (2017). Smoking may lead to marginal bone loss around non-submerged implants during bone healing by altering salivary microbiome: a prospective study. J. Periodontol. 88, 1297-1308. doi: 10.1902/jop.2017.160808

Fine, D. H., Markowitz, K., Fairlie, K., Tischio-Bereski, D., Ferrendiz, J., Furgang, D., et al. (2013). A consortium of Aggregatibacter actinomycetemcomitans (Aa), Streptococcus parasanguinis and Filifactor alocis are present in sites prior to bone loss in a longitudinal study of localized aggressive periodontitis. J. Clin. Microbiol. 51, 2850-2861. doi: 10.1128/jcm.00729-13

George, N., Flamiatos, E., Kawasaki, K., Kim, N., Carriere, C., Phan, B., et al. (2016). Oral microbiota species in acute apical endodontic abscesses. J. Oral Microbiol. 8:30989. doi: 10.3402/jom.v8.30989

Herrera, D., Alonso, B., de Arriba, L., Santa Cruz, I., Serrano, C., and Sanz, M. (2014). Acute periodontal lesions. Periodontol 65, 149-177. doi: 10.1111/prd. 12022

Herrera, D., Roldan, S., and Sanz, M. (2000). The periodontal abscess: a review. J. Clin. Periodontol. 27, 377-386. doi: 10.1034/j.1600-051x.2000.02700 6377.x
Jaramillo, A., Arce, R. M., Herrera, D., Betancourth, M., Botero, J. E., and Contreras, A. (2005). Clinical and microbiological characterization of periodontal abscesses. J. Clin. Periodontol. 32, 1213-1218. doi: 10.1111/j.1600051X.2005.00839.x

Jorth, P., Turner, K. H., Gumus, P., Nizam, N., Buduneli, N., and Whiteley, M. (2014). Metatranscriptomics of the human oral microbiome during health and disease. mBio 5, e01012-e01014. doi: 10.1128/mBio.01012-14

Kozarov, E. V., Dorn, B. R., Shelburne, C. E., Dunn, W. A. Jr., and Progulske-Fox, A. (2005). Human atherosclerotic plaque contains viable invasive Actinobacillus actinomycetemcomitans and Porphyromonas gingivalis. Arterioscler. Thromb. Vasc. Biol. 25, e17-e18. doi: 10.1161/01.ATV.0000155018.67835.1a

Kumar, P., Griffen, A., Barton, J., Paster, B., Moeschberger, M., and Leys, E. (2003). New bacterial species associated with chronic periodontitis. J. Dent. Res. 82, 338-344. doi: 10.1177/154405910308200503

Liu, B., Faller, L. L., Klitgord, N., Mazumdar, V., Ghodsi, M., Sommer, D. D., et al. (2012). Deep sequencing of the oral microbiome reveals signatures of periodontal disease. PLoS One 7:e37919. doi: 10.1371/journal.pone.0037919

Lozupone, C., Lladser, M. E., Knights, D., Stombaugh, J., and Knight, R. (2011). UniFrac: an effective distance metric for microbial community comparison. ISME J. 5, 169-172. doi: 10.1038/ismej.2010.133

Mizrahi-Man, O., Davenport, E. R., and Gilad, Y. (2013). Taxonomic classification of bacterial 16S rRNA genes using short sequencing reads: evaluation of effective study designs. PLoS One 8:e53608. doi: 10.1371/journal.pone.0053608

Moffatt, C. E., Whitmore, S. E., Griffen, A. L., Leys, E. J., and Lamont, R. J. (2011). Filifactor alocis interactions with gingival epithelial cells. Mol. Oral Microbiol. 26, 365-373. doi: 10.1111/j.2041-1014.2011.00624.x

Newman, M. G., and Sims, T. N. (1979). The predominant cultivable microbiota of the periodontal abscess. J. Periodontol. 50, 350-354. doi: 10.1902/jop.1979.50. 7.350

Newman, M. G., Takei, H. H., and Klokkevold, P. R. (2015). Carranza’s Clinical Periodontology, 12th Edn. St. Louis, MI: Elsevier.

Paju, S., Oittinen, J., Haapala, H., Asikainen, S., Paavonen, J., and Pussinen, P. J. (2017). Porphyromonas gingivalis may interfere with conception in women. J. Oral Microbiol. 9:1330644. doi: 10.1080/20002297.2017.1330644

Saito, A., Sojar, H. T., and Genco, R. J. (1997). Interleukin-1 gene expression in macrophages induced by surface protein components of Porphyromonas gingivalis: role of tyrosine kinases in signal transduction. Oral Microbiol. Immunol. 12, 135-140. doi: 10.1111/j.1399-302x.1997.tb00369.x

Sato, J., Kuroshima, T., Wada, M., Satoh, A., Watanabe, S., Okamoto, S., et al. (2016). Use of FDG-PET to detect a chronic odontogenic infection as a possible source of the brain abscess. Odontology 104, 239-243. doi: 10.1007/s10266-0150218-211

Schlafer, S., Riep, B., Griffen, A. L., Petrich, A., Hubner, J., Berning, M., et al. (2010). Filifactor alocis-involvement in periodontal biofilms. BMC Microbiol. 10:66. doi: 10.1186/1471-2180-10-66

Schloss, P. D., Westcott, S. L., Ryabin, T., Hall, J. R., Hartmann, M., Hollister, E. B., et al. (2009). Introducing mothur: open-source, platform-independent, community-supported software for describing and comparing microbial communities. Appl. Environ. Microbiol. 75, 7537-7541. doi: 10.1128/AEM. 01541-09

Smith, R., and Davies, R. (1986). Acute lateral periodontal abscesses. Br. Dent. J. 161, 176-178. doi: 10.1038/sj.bdj.4805920

Socransky, S. S., Haffajee, A. D., Cugini, M. A., Smith, C., and Kent, R. L. (1998). Microbial complexes in subgingival plaque. J. Clin. Periodontol. 25, 134-144. doi: 10.1111/j.1600-051x.1998.tb02 419.x

Tennert, C., Fuhrmann, M., Wittmer, A., Karygianni, L., Altenburger, M. J., Pelz, K., et al. (2014). New bacterial composition in primary and persistent/secondary endodontic infections with respect to clinical and radiographic findings. J. Endod. 40, 670-677. doi: 10.1016/j.joen.2013.10.005

Wang, J., Qi, J., Zhao, H., He, S., Zhang, Y., Wei, S., et al. (2013). Metagenomic sequencing reveals microbiota and its functional potential associated with periodontal disease. Sci. Rep. 3:1843. doi: 10.1038/srep01843

Webb, G. J., Chapman, T. P., Cadman, P. J., and Gorard, D. A. (2014). Pyogenic liver abscess. Frontline Gastroenterol. 5, 60-67. doi: 10.1136/flgastro-2013100371

WHO (1997). Oral Health Surveys: Basic Methods, 4th Edn. Geneva: World Health Organization 
Wu, X., Chen, J., Xu, M., Zhu, D., Wang, X., Chen, Y., et al. (2017). 16S rDNA analysis of periodontal plaque in chronic obstructive pulmonary disease and periodontitis patients. J. Oral Microbiol. 9:1324725. doi: 10.1080/20002297. 2017.1324725

Xie, Y., Chen, J., He, J., Miao, X., Xu, M., Wu, X., et al. (2014). Antimicrobial resistance and prevalence of resistance genes of obligate anaerobes isolated from periodontal abscesses. J. Periodontol. 85, 327-334. doi: 10.1902/jop.2013. 130081

Xu, L., Chen, X., Wang, Y., Jiang, W., Wang, S., Ling, Z., et al. (2018). Dynamic alterations in salivary microbiota related to dental caries and age in preschool children with deciduous dentition: a 2-Year follow-up study. Front. Physiol. 9:342. doi: $10.3389 /$ fphys. 2018.00342

Yazbeck, M. F., Dahdel, M., Kalra, A., Browne, A. S., and Pratter, M. R. (2014). Lung abscess: update on microbiology and management. Am. J. Ther. 21, 217-221. doi: 10.1097/MJT.0b013e3182383c9b
Yoneda, M., Kato, S., Mawatari, H., Kirikoshi, H., Imajo, K., Fujita, K., et al. (2011). Liver abscess caused by periodontal bacterial infection with Fusobacterium necrophorum. Hepatol. Res. 41, 194-196. doi: 10.1111/j.1872-034X.2010.00 748.x

Conflict of Interest Statement: The authors declare that the research was conducted in the absence of any commercial or financial relationships that could be construed as a potential conflict of interest.

Copyright $\odot 2019$ Chen, $W u, Z h u, X u, Y u, Y u$ and Zhang. This is an open-access article distributed under the terms of the Creative Commons Attribution License (CC BY). The use, distribution or reproduction in other forums is permitted, provided the original author(s) and the copyright owner(s) are credited and that the original publication in this journal is cited, in accordance with accepted academic practice. No use, distribution or reproduction is permitted which does not comply with these terms. 